\title{
Linear and nonlinear universality in the rheology of polymer melts and solutions
}

\author{
Wingstrand, Sara Lindeblad; Alvarez, Nicolas J.; Huang, Qian; Hassager, Ole
}

Published in:

Physical Review Letters

Link to article, DOI:

10.1103/physrevlett.115.078302

Publication date:

2015

Document Version

Publisher's PDF, also known as Version of record

Link back to DTU Orbit

Citation (APA):

Wingstrand, S. L., Alvarez, N. J., Huang, Q., \& Hassager, O. (2015). Linear and nonlinear universality in the rheology of polymer melts and solutions. Physical Review Letters, 115(7), [078302].

https://doi.org/10.1103/physrevlett.115.078302

\section{General rights}

Copyright and moral rights for the publications made accessible in the public portal are retained by the authors and/or other copyright owners and it is a condition of accessing publications that users recognise and abide by the legal requirements associated with these rights.

- Users may download and print one copy of any publication from the public portal for the purpose of private study or research.

- You may not further distribute the material or use it for any profit-making activity or commercial gain

- You may freely distribute the URL identifying the publication in the public portal

If you believe that this document breaches copyright please contact us providing details, and we will remove access to the work immediately and investigate your claim 


\title{
Linear and Nonlinear Universality in the Rheology of Polymer Melts and Solutions
}

\author{
Sara L. Wingstrand, ${ }^{1}$ Nicolas J. Alvarez, ${ }^{2}$ Qian Huang, ${ }^{1}$ and Ole Hassager ${ }^{1, *}$ \\ ${ }^{1}$ Danish Polymer Center, Department of Chemical and Biochemical Engineering, Technical University of Denmark, \\ Building 227, 2800 Kgs. Lyngby, Denmark \\ ${ }^{2}$ Department of Chemical and Biological engineering, Drexel University, Philadelphia, Pennsylvania 19104, USA
}

(Received 5 May 2015; published 13 August 2015)

\begin{abstract}
Understanding the dynamics of polymeric liquids has great importance in the design and processing of soft materials. While slow flow dynamics is now resolved, fast flow dynamics is still unsolved, especially due to the lack of experimental evidence. We here manipulate a poly(methyl methacrylate) solution into exhibiting the same flow behavior as a polystyrene melt. Strikingly similar responses of the fluids are seen both in slow and very fast flow. With this discovery we show that dynamics in polymeric liquids can be generalized and captured in one single polymer physics model.
\end{abstract}

DOI: 10.1103/PhysRevLett.115.078302

Universality of molecular dynamics is a fundamental assumption in polymer physics [1,2]. It is the underlying framework of the most successful theoretical model, known as the tube model $[3,4]$. The significance of this assumption is that model systems at room temperature, i.e., polymer solutions, could be used to represent polymer melts at elevated temperatures. While the existence of a room temperature model material would be highly desirable, unfortunately a polymer solution with the same fast flow dynamics (nonlinear behavior) as a polymer melt has not yet been observed. It was recently shown that two linear polymeric systems with the same number of entanglements exhibit identical slow flow dynamics (linear behavior), but strikingly different nonlinear behavior [5]. The lack of evidence of universality in strongly nonlinear conditions leaves one wondering whether such an assumption is valid.

For two polymeric systems to have identical flow dynamics, researchers have hypothesized that they must have the same following characteristics: (i) the same number of entanglements, (ii) the same degree of flexibility (number of Kuhn segments per entanglement), and, very recently, (iii) the same potential for monomeric friction reduction [6]. Whereas systems with identical characteristics (i) and (ii) have been studied [7,8], systems with the same characteristics (i)-(iii) have not, the reason being that usually characteristic (ii) can never be adjusted without compromising characteristic (iii).

This study observes universal behavior between a polymer solution and a polymer melt with the same three characteristics, confirming the assumption of universality in polymer physics for both linear and nonlinear dynamics. The following is a brief description of how to manipulate all three characteristics independently.

Adjustment of the three characteristics (i)-(iii) is based on the tube model depicted in Fig. 1. Here, a polymer in an entangled melt is reduced to a primitive chain with limited ability to move in its transverse direction due to
PACS numbers: 47.57.Ng, 83.10.Kn, 83.60.Bc, 83.60.Df

entanglements with neighboring chains [Fig. 1(a)]. Effectively, the entanglements can be regarded as constituents of a tube surrounding the test chain [Fig. 1(b)]. The primitive chain itself can be described by a random walk of Kuhn steps [Fig. 1(c)].

The number of entanglements per chain $Z$, characteristic (i), is solely responsible for the linear response of a polymeric liquid [5]. It is given by the ratio of Kuhn steps in the entire chain $N$ over Kuhn steps per entanglement segment $N_{e}$, or analogously on a molar mass basis

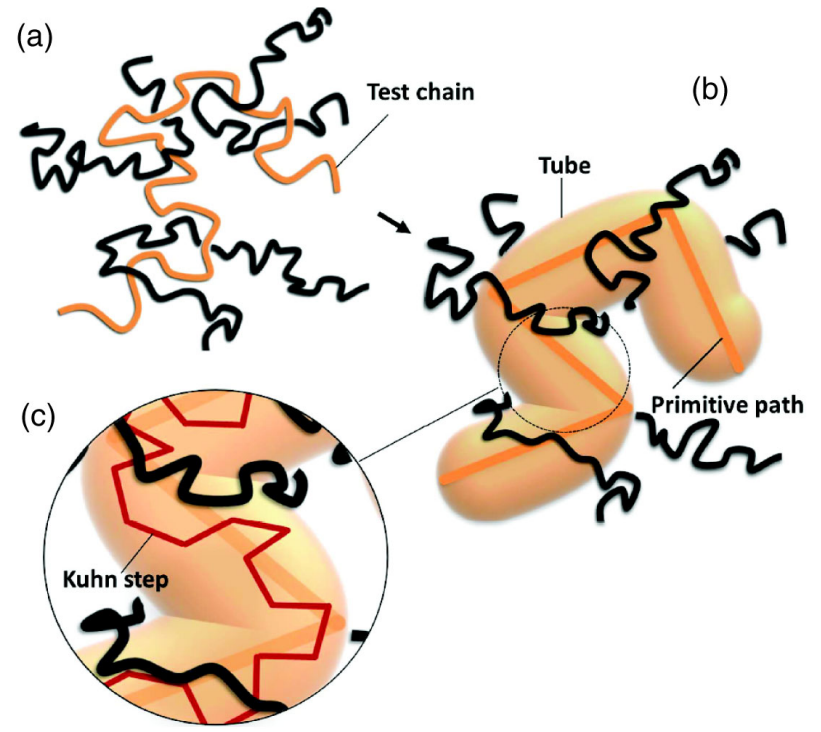

FIG. 1 (color online). Sketch of the tube analogy for entangled polymeric liquids. (a) A primitive entangled polymer chain in a melt. The polymer test chain is well entangled with neighboring polymer chains. (b) The simplified picture of the polymer melt applied in the original tube model. Here, the entanglements constitute a tube with a primitive path. (c) Enlargement of one entanglement segment showing how the test chain is reduced to a random distribution of connected Kuhn steps. 


$$
Z=\frac{N}{N_{e}}=\frac{M_{w}}{M_{e}} .
$$

Here, $M_{w}$ and $M_{e}$ are the molar masses of the entire chain and one entanglement segment, respectively. As $M_{e}$ is independent of polymer molar mass, the number of entanglements can be adjusted from the molar mass of the polymer as it scales linearly with $M_{w}$. The addition of a solvent to a polymer melt increases $M_{e}$. However, a proportional change in $M_{w}$ may be used to keep the number of entanglements unchanged.

The number of Kuhn steps per entanglement $N_{e}$, characteristic (ii), becomes important in the nonlinear regime [9]. $N_{e}$ describes the rigidity of an entanglement segment and is used in several nonlinear models [10-15]. For an undiluted polymer, $N_{e}$ is determined by its chemistry alone. Adding a solvent to an entangled polymeric liquid will increase the spacing between entanglements, thus increasing the number of Kuhn steps per entanglement segment. In a solution with a given polymer concentration $\phi$ the number of Kuhn steps between entanglements $N_{e}(\phi)$ scales as

$$
N_{e}(\phi)=N_{e}(1) \phi^{-\alpha} .
$$

Here, $N_{e}(1)$ is the number of Kuhn steps per entanglement segment for the undiluted system and $\alpha$ is the dilution exponent with a value between 1 and 1.3, depending on concentration [16]. It is realized that dilution increases $N_{e}$; hence, the only way to match $N_{e}$ between a polymer melt and a solution is to have two different chemistries. Thus, by allowing a change in chemistry and molecular weight, both $N_{e}$ and $Z$ can be adjusted independently.

As previously mentioned, no two polymer liquids having identical characteristics (i) and (ii) (i.e., the same $N_{e}$ and Z) have shown the same nonlinear behavior. In fact, evidence of the contrary is available [7]. Hence, experimental evidence suggests that $Z$ and $N_{e}$ alone cannot account for the nonlinear behavior of polymeric liquids. This is why the third concept, monomeric friction reduction, proposed by Ianniruberto and co-workers is important [6].

Friction reduction, characteristic (iii), encountered in nonlinear flows, arises from the anisotropic environment locally around the polymer chain [6,17]. In the case of diluted polymers, traditional (small) solvent molecules remain isotropic even at large deformations, inhibiting any flow-induced monomeric friction reduction. The very nature of friction reduction seemingly disrupts any possibility of ever obtaining a solution that behaves as a melt, unless the solvent molecule possesses the same potential for friction reduction as the polymer itself, i.e., such as in the case of oligomers.

Using oligomers (polymers with less than 100 repeating units) as the solvent is a potential method of matching the friction reduction between the polymer and the solvent. Molecular dynamics simulations of polystyrene oligomers have shown that the degree of friction reduction in fast shear flows indeed increases with increasing molar mass of the oligomers [6]. Hence, we hypothesize that the friction reduction of a polymer solution increases as the number of oligomer Kuhn steps $N_{s}$ increases. Yet, in order not to create a binary blend of polymers where $N_{e}$ is fixed, $N_{s}$ must be smaller than $N_{e}$ to avoid forming any additional entanglements, i.e., $N_{s} / N_{e}<1$. On the contrary $N_{s} / N_{e}$ should be as large as possible in order to have a similar potential for anisotropy and hence friction reduction as a melt.

The solutions investigated in this study are prepared from poly(methyl methacrylate) (PMMA) with $M_{w}=86 \mathrm{~kg} / \mathrm{mol}$ (PMMA-86k) and $M_{w}=270 \mathrm{~kg} / \mathrm{mol}$ (PMMA-270k). From linear characterization, $Z$ is estimated to be 14 for PMMA-86k and 52 for PMMA-270k, see the Supplemental Material [18] for details. $N_{e}$ is estimated to be 10 and 9, respectively [19]. Oligo(methyl methacrylates) of various lengths are investigated as the solvent for the samples. Among the various options (see the Supplemental Material [18]), an oligomer of $M_{w}=$ $3.5 \mathrm{~kg} / \mathrm{mol}$ (referred to as $\mathrm{o}-4 \mathrm{k}$ ) is found to be the most optimal solvent for this study, i.e., the longest solvent molecule that does not form entanglements.

The reference materials that we aim to mimic using PMMA are two polystyrene (PS) melts. They have previously been characterized by Nielsen et al. and Huang et al. [5,21], one with $M_{w}$ equal to $103 \mathrm{~kg} / \mathrm{mol}$ the other with $285 \mathrm{~kg} / \mathrm{mol}$, referred to as PS-100k and PS-285k, respectively. PS is known to have $M_{e}=13.3 \mathrm{~kg} / \mathrm{mol}$ and analogously $N_{e}=22[5,22]$. Characteristics of these samples related to the tube model are given in Table I.

Diluting PMMA-86k and PMMA-270k in o-4k yields the solutions PMMA-86k/o-4k and PMMA-270k/o-4k, respectively, with the characteristics given in Table I. Values of $Z$ and $N_{e}$ for the PMMA solutions and the reference PS melts are in good agreement (within $\pm 10 \%$ ). Furthermore, we observe that $N_{s} / N_{e}$ for the PMMA solutions are close to 1 without exceeding it, as required.

The linear and nonlinear response of the PMMA solutions and PS melts are shown in Figs. 2(a) and 2(b), respectively. The linear response is obtained from a small amplitude oscillatory shear (SAOS) flow whereas nonlinear characterization is obtained from uniaxial extension.

TABLE I. Material characteristics of PS melts and PMMA solutions.

\begin{tabular}{lccccc}
\hline \hline Sample & $\phi$ & $\alpha$ & $Z$ & $N_{e}$ & $N_{S} / N_{e}$ \\
\hline PS-285k & 1.00 & $\ldots$ & 21 & 22 & $\ldots$ \\
PMMA-270k/o-4k & 0.45 & 1.17 & 20 & 23 & 0.67 \\
PS-100k & 1.00 & $\ldots$ & 7.7 & 22 & $\ldots$ \\
PMMA-86k/o-4k & 0.51 & 1.08 & 7.0 & 20 & 0.59 \\
\hline \hline
\end{tabular}



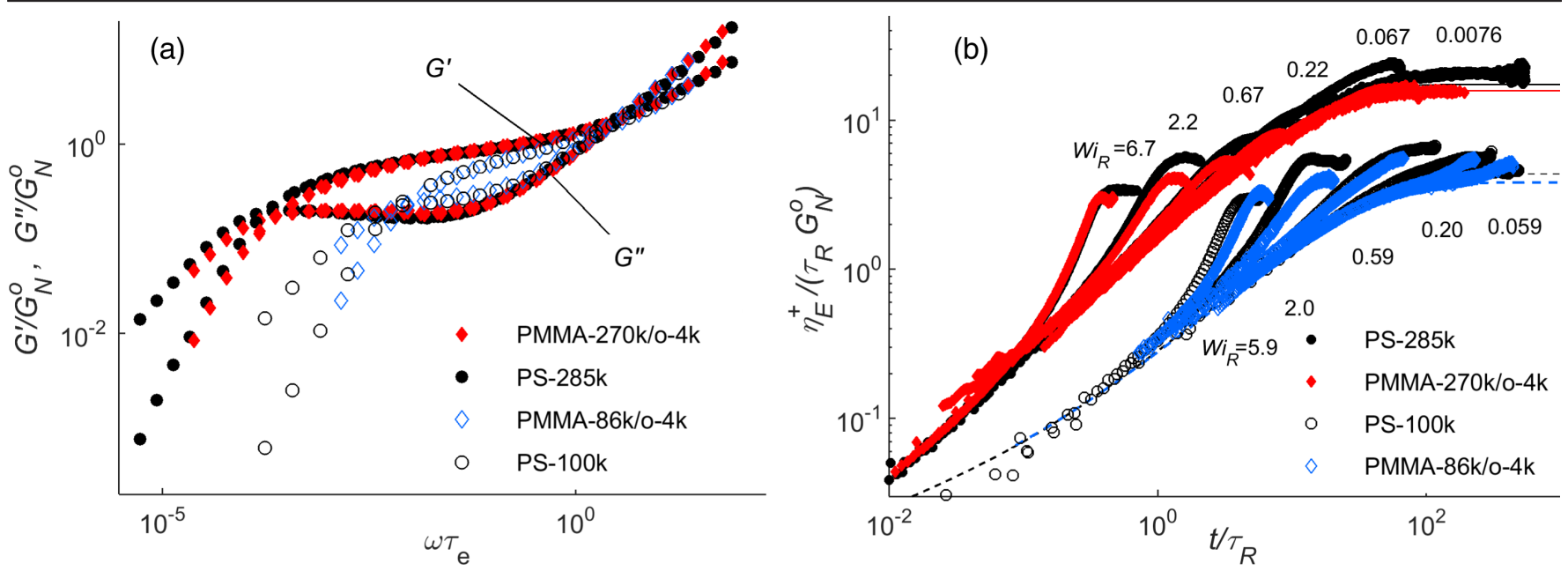

FIG. 2 (color online). Nondimensional mechanical response of PS melts and PMMA solutions. Samples have a similar number of Kuhn steps between entanglements $N_{e}$, degree of friction reduction, and pairwise matching number of entanglements $Z$. Closed symbols indicate samples with $Z \approx 20$, open symbols samples with $Z \approx 7$. Black indicates reference data for pure polystyrene melts [5,21]. (a) Linear response from small amplitude oscillatory shear, expressed in terms of normalized dynamic moduli versus normalized frequency. (b) Response of melts and solutions in extensional flow at various nondimensional rates of deformation (Wi $\mathrm{i}_{R}$ ). Results are expressed in terms of the normalized stress growth coefficient. Samples with $\mathrm{Z} \approx 7$ have been shifted horizontally one order of magnitude higher for clarity. Solid and dashed lines are linear predictions obtained from fits of the SAOS data.

The linear response [Fig. 2(a)] is expressed in terms of the dynamic moduli $G^{\prime}$ and $G^{\prime \prime}$ versus frequency $\omega$, representing the elastic and viscous response of the fluid, respectively. The solvent is expected to contribute an amount to $G^{\prime \prime}=(1-\phi) \eta_{s} \omega$, where $\eta_{s}=410 \mathrm{Pas}$ is the solvent viscosity. Hence, the solvent only contributes a negligible amount to the moduli and the solvent does not affect the universality argument. The dynamic moduli have been normalized by the characteristic plateau modulus $G_{N}^{0}$, a hypothetical value of the plateau in $G^{\prime}$ for $\omega \rightarrow \infty$. Frequency is normalized by the characteristic time $\tau_{e}$ related to the second crossover of the dynamic moduli. The parameters $G_{N}^{0}, \tau_{e}$, and $Z$ may be extracted from models such as the Baumgaertel-Schausberger-Winter spectrum or the Likhtman-Mcleish model (see the Supplemental Material [18]) [23,24].

From the linear characterization in Fig. 2(a), it is seen how samples with the same $Z$ overlap, as expected. Tube stretch and friction reduction are inactive under these conditions and, consequently, $N_{e}$ as well as the type of solvent are irrelevant for the observed similarity.

The most severe nonlinear behavior of polymers is encountered in extension, including uniaxial extension. This study employs a filament stretching rheometer to measure the fast flow dynamics of melts and solutions [25]. It is equipped with an active control scheme [26] to avoid filament instability as described by Fielding [27]. The instrument measures the stress in the polymer sample by monitoring the axial force and the midfilament diameter. This study performs extensional measurements at a controlled rate, the Hencky strain rate $\dot{\varepsilon}_{0}$. The resulting response of the polymeric liquids is shown in Fig. 2(b). It displays the transient behavior in terms of the extensional stress growth coefficient, given by

$$
\eta_{E}^{+}(t)=\frac{\sigma_{z z}-\sigma_{r r}}{\dot{\varepsilon}_{0}}
$$

Here, $\sigma_{z z}$ and $\sigma_{r r}$ represent the axial and radial component of the stress tensor, respectively. Normalization of $\eta_{E}^{+}$ is based on a characteristic viscosity given by the plateau modulus $G_{N}^{0}$ and a characteristic relaxation time scale for the whole polymer chain $\tau_{R}=\tau_{e} Z^{2}$ (see the Supplemental Material [18] for more details). In addition, the linear prediction obtained from fitting the BaumgaertelSchausberger-Winter spectrum to the SAOS data is shown as solid and dashed lines in Fig. 2(b).

Each upturn represents a single filament stretch experiment, performed at a given constant $\dot{\varepsilon}_{0}$. To enable comparison, strain rates are given in terms of nondimensional Weissenberg numbers $\left(\mathrm{Wi}_{R}=\dot{\varepsilon}_{0} \tau_{R}\right.$ indicated at each experiment) instead of the absolute value $\dot{\varepsilon}_{0}$. $\mathrm{Wi}_{R}$ compares the Rouse relaxation time of the chain contour length $\tau_{R}$ to the imposed rate of deformation $\dot{\varepsilon}_{0}$ [28]. As long as $\mathrm{Wi}_{R}<1$ the number of Kuhn lengths between entanglements $N_{e}$ is unimportant. However, for $\mathrm{Wi}_{R}>1$ motions on the scale of a Kuhn length occur, and therefore $N_{e}$ becomes an important parameter. In a similar way friction reduction is only activated in a highly anisotropic environment experienced at $\mathrm{Wi}_{R}>1$, where chains are aligned and stretched [29]. 


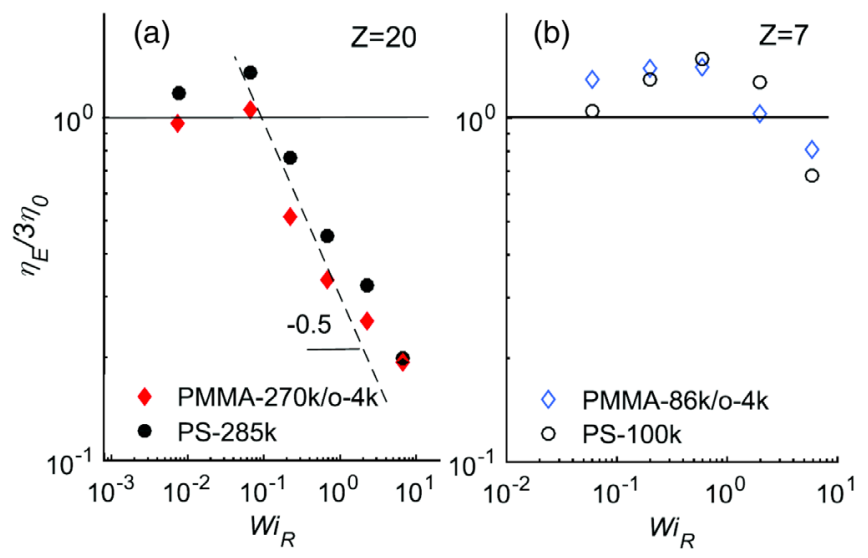

FIG. 3 (color online). Normalized steady state viscosity as a function of the Weissenberg number. Panel (a) compares samples with $Z \approx 20$. Panel (b) compares samples with $Z \approx 7$. Black indicates reference data $[5,21]$.

In Fig. 2(b) good agreement with the linear prediction is observed for $\mathrm{Wi}_{R} \ll 1$, as expected. At larger $\mathrm{Wi}_{R}$ a clear deviation from linear behavior is observed. This upward deviation of $\eta_{E}^{+}$from the linear prediction is known as strain hardening. The most important characteristic is that the transient behavior of PS melts and PMMA solutions stretched at the same $\mathrm{Wi}_{R}$ is the same for all time, for all experiments. This similarity between melt and solution behavior has, to our knowledge, never been observed before.

A plateau region is observed for $\eta_{E}^{+}$, towards the end of each stretch experiment in Fig. 2(b). Here, the polymeric liquids reach a steady state extensional viscosity $\eta_{E}$. These values are plotted against $\mathrm{Wi}_{R}$ in Fig. 3. In the linear regime, extensional viscosity $\eta_{E}$ is often expressed in terms of the zero shear rate viscosity $\eta_{0}$, where $\eta_{E}=3 \eta_{0}$. Theoretically, $\eta_{E} / 3 \eta_{0} \rightarrow 1$ as $\mathrm{Wi}_{R} \rightarrow 0$ for linear polymeric liquids, as is indeed the case here.

The PMMA solutions prepared in this work exhibit an initial increase in $\eta_{E}$, followed by a steady decrease. The increase is more pronounced for a low number of entanglements both for the solutions and the melts. Furthermore, for liquids with a sufficiently high number of entanglements, $\eta_{E}$ follows the power law $\eta_{E} \propto\left(\mathrm{Wi}_{R}\right)^{-0.5}$, previously only observed for polymer melts [30]. Overall, the PMMA solutions and PS melts with the same three characteristics behave identically.

The ability of the oligomer to induce friction reduction can be explained from the concept of average orientation introduced by Yaoita et al. [17]. Assuming an isotropic solvent, they model friction reduction by introducing an average orientation tensor $\overline{\boldsymbol{S}}=\phi \boldsymbol{S}_{p}$ with $\boldsymbol{S}_{p}$ being the polymer orientation tensor. The greater the average orientation, the greater the friction reduction. It is seen that the average orientation clearly is reduced as the concentration of polymer $\phi$ is reduced, and consequently the degree of friction is reduced.

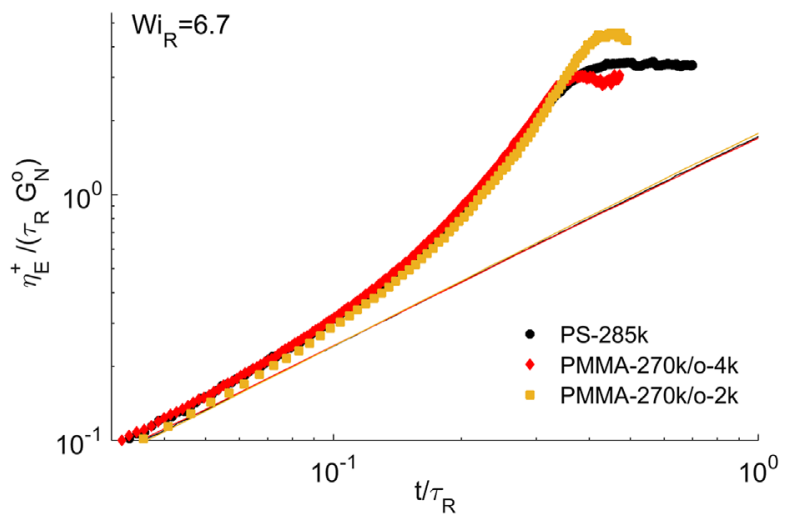

FIG. 4 (color online). Normalized stress growth coefficient (symbols) for three different polymers extended at a Weissenberg number $\mathrm{Wi}_{R}=6.7$. Solid lines indicate the predicted linear response. Black indicates reference data [5].

In this work, the o- $4 \mathrm{k}$ solvent is orientable, i.e., nonisotropic. Intuitively, there must be an additional contribution to the average orientation from the solvent such that $\overline{\boldsymbol{S}}=\phi \boldsymbol{S}_{p}+(1-\phi) \boldsymbol{S}_{s}$ where $\boldsymbol{S}_{s}$ is the orientation tensor of the solvent. Therefore, the average orientation of the solution is higher than if a molecular (isotropic) solvent were used, promoting friction reduction as expected in a melt. Note that in the limiting case where $\boldsymbol{S}_{s} \approx \boldsymbol{S}_{p}$ there should be little difference between a melt and a solution.

To demonstrate this concept further, the effect of using a solvent molecule with $M_{w}=2.1 \mathrm{~kg} / \mathrm{mol}$ (o-2k), i.e., half the size of the utilized o-4k, is seen in Fig. 4. It is seen that the steady state viscosity of the PMMA solution containing the o-2k solvent (PMMA-270k/o-2k) exceeds that of the two others, PMMA-270k/o-4k and PS-285k. This supports the hypothesis that the o-2k solvent contributes less to the average orientation than the $0-4 \mathrm{k}$, resulting in less friction reduction.

To conclude, we have shown experimentally that universality of polymer dynamics can be extended from the linear regime deep into the nonlinear regime. The concept of monomeric friction reduction seems valid and perfectly explains the previously unsolved discrepancy between the response of polymer melts and solutions in fast flows where both $Z$ and $N_{e}$ are the same. As a result the number of characteristics needed to fully describe flows of polymeric liquids across all deformation regimes can be narrowed down to the three presented here.

These results have both positive and negative implications, the positive being that we now have a method of systematically designing model materials for linear entangled polymers and most likely other macromolecules and polymers with other types of architectures due to the proof of universality [31]. Unfortunately, the influence of friction reduction means that diluting polymers with conventional molecular solvents can never result in a proper model material for a polymeric melt since a disparity in 
friction reduction between the polymer and solvent increases the strain hardening behavior. Hence, the use of polymeric solutions as model materials for polymeric melts seems very limited. The important take home message is that this data set offers a complete experimental framework for which to test all future models, which evidently must include physics relating to the number of entanglements, the flexibility of the chain, and the monomeric friction reduction of both the polymer and solvent when applicable.

In closing we remark that universality has been demonstrated on the macroscopically observable stress. It is interesting to consider whether this reflects universality on the molecular level, which in the future could be observed by techniques such as dielectric spectroscopy or neutron scattering $[32,33]$.

Please contact Ole Hassager to gain access to the raw data.

We thank Evelyne van Ruymbeke at Université Catholique de Louvain for motivating the field of study. Financial support from the Aage og Johanne Louis-Hansen Foundation and the European Union (SUPOLEN, Initial Training Networks) is gratefully acknowledged.

*oh@kt.dtu.dk

[1] D. J. Read, D. Auhl, C. Das, J. den Doelder, M. Kapnistos, I. Vittorias, and T.C.B. McLeish, Science 333, 1871 (2011).

[2] M. W. Collis, A. K. Lele, M. R. Mackley, R. S. Graham, D. J. Groves, A. E. Likhtman, T. M. Nicholson, T. C. B. Mcleish, L. R. Hutchings, C. M. Fernyhough, and R. N. Young, J. Rheol. 49, 501 (2005).

[3] P. G. de Gennes, J. Chem. Phys. 55, 572 (1971).

[4] M. Doi and S.F. Edwards, The Theory of Polymer Dynamics (Clarendon, Oxford, 1986), p. 391.

[5] Q. Huang, O. Mednova, H. K. Rasmussen, N. J. Alvarez, A. L. Skov, and O. Hassager, Macromolecules 46, 5026 (2013).

[6] G. Ianniruberto, A. Brasiello, and G. Marrucci, Macromolecules 45, 8058 (2012).

[7] Q. Huang, N. J. Alvarez, Y. Matsumiya, H. K. Rasmussen, H. Watanabe, and O. Hassager, ACS Macro Lett. 2, 741 (2013).

[8] T. Sridhar, M. Acharya, D. a. Nguyen, and P. K. Bhattacharjee, Macromolecules 47, 379 (2014).

[9] C. Luap, C. Müller, T. Schweizer, and D. C. Venerus, Rheol. Acta 45, 83 (2005).

[10] J. Fang, M. Kröger, and H. C. Öttinger, J. Rheol. 44, 1293 (2000).
[11] H. K. Rasmussen and Q. Huang, J. Non-Newtonian Fluid Mech. 204, 1 (2014).

[12] T. Yaoita, T. Isaki, Y. Masubuchi, H. Watanabe, G. Ianniruberto, and G. Marrucci, Macromolecules 44, 9675 (2011).

[13] E. van Ruymbeke, J. Nielsen, and O. Hassager, J. Rheol. 54, 1155 (2010).

[14] R. S. Graham, A. E. Likhtman, T. C. B. McLeish, and S. T. Milner, J. Rheol. 47, 1171 (2003).

[15] D. J. Read, K. Jagannathan, S. K. Sukumaran, and D. Auhl, J. Rheol. 56, 823 (2012).

[16] E. van Ruymbeke, Y. Masubuchi, and H. Watanabe, Macromolecules 45, 2085 (2012).

[17] T. Yaoita, T. Isaki, Y. Masubuchi, H. Watanabe, G. Ianniruberto, and G. Marrucci, Macromolecules 45, 2773 (2012).

[18] See Supplemental Material at http://link.aps.org/ supplemental/10.1103/PhysRevLett.115.078302 for further information regarding materials and method, data fitting and extracted material parameters along with raw data.

[19] Values differ between the two PMMAs due to a difference in tacticity [20].

[20] K. Fuchs, C. Friedrich, and J. Weese, Macromolecules 29, 5893 (1996).

[21] J. K. Nielsen, H. K. Rasmussen, O. Hassager, and G. H. McKinley, J. Rheol. 50, 453 (2006).

[22] L. J. Fetters, D. J. Lohse, and R. H. Colby, in Physical Properties of Polymers Handbook, edited by J. E. Mark, 2nd ed. (Springer, New York, 2007), p. 1073.

[23] M. Baumgaertel, A. Schausberger, and H. H. Winter, Rheol. Acta 29, 400 (1990).

[24] A. E. Likhtman and T. C. B. McLeish, Macromolecules 35, 6332 (2002).

[25] A. Bach, H. Koblitz, P. Longin, and O. Hassager, J. Non-Newtonian Fluid Mech. 108, 163 (2002).

[26] J. M. Román Marín, J. K. Huusom, N. J. Alvarez, Q. Huang, H. K. Rasmussen, A. Bach, A. L. Skov, and O. Hassager, J. Non-Newtonian Fluid Mech. 194, 14 (2013).

[27] S. M. Fielding, Phys. Rev. Lett. 107, 258301 (2011).

[28] J. M. Dealy, Rheol. Bull. 79, 14 (2010).

[29] O. Hassager, K. Mortensen, A. Bach, K. Almdal, H. K. Rasmussen, and W. Pyckhout-Hintzen, Rheol. Acta 51, 385 (2012).

[30] A. Bach, K. Almdal, H. K. Rasmussen, and O. Hassager, Macromolecules 36, 5174 (2003).

[31] R. G. Larson, Science 318, 57 (2007).

[32] K. Horio, T. Uneyama, Y. Matsumiya, Y. Masubuchi, and H. Watanabe, Macromolecules 47, 246 (2014).

[33] A. Blanchard, R. S. Graham, M. Heinrich, W. PyckhoutHintzen, D. Richter, A. E. Likhtman, T. C. B. McLeish, D. J. Read, E. Straube, and J. Kohlbrecher, Phys. Rev. Lett. 95, 166001 (2005). 